\title{
A Comparative Study of Three Different Mathematical Methods for Solving the Unit Commitment Problem
}

\author{
Mehmet Kurban and Ümmühan Başaran Filik \\ Department of Electrical and Electronics Engineering, Anadolu University, 26470 Eskişehir, Turkey \\ Correspondence should be addressed to Mehmet Kurban, mkurban@anadolu.edu.tr
}

Received 23 December 2008; Revised 10 March 2009; Accepted 10 March 2009

Recommended by Joaquim J. Júdice

\begin{abstract}
The unit commitment (UC) problem which is an important subject in power system engineering is solved by using Lagragian relaxation (LR), penalty function (PF), and augmented Lagrangian penalty function (ALPF) methods due to their higher solution quality and faster computational time than metaheuristic approaches. This problem is considered to be a nonlinear programming(NP-) hard problem because it is nonlinear, mixed-integer, and nonconvex. These three methods used for solving the problem are based on dual optimization techniques. ALPF method which combines the algorithmic aspects of both LR and PF methods is firstly used for solving the UC problem. These methods are compared to each other based on feasible schedule for each stage, feasible cost, dual cost, duality gap, duration time, and number of iterations. The numerical results show that the ALPF method gives the best duality gap, feasible and dual cost instead of worse duration time and the number of iterations. The four-unit Tuncbilek thermal plant which is located in Kutahya region in Turkey is chosen as a test system in this study. The programs used for all the analyses are coded and implemented using general algebraic modeling system (GAMS).
\end{abstract}

Copyright (c) 2009 M. Kurban and Ü. Başaran Filik. This is an open access article distributed under the Creative Commons Attribution License, which permits unrestricted use, distribution, and reproduction in any medium, provided the original work is properly cited.

\section{Introduction}

The UC problem decides to which electricity generation units should be running in each stage to satisfy a predictably varying demand for electricity. The solution of the UC problem is a complex optimization problem, and UC problem can be considered as two linked optimization problems: the first is a combinatorial optimization problem, and the second is a nonlinear programming problem. Due to important startup and shutdown costs, the problem is in general very hard to solve, as it is not possible to perform a separate optimization for each time interval. The exact solution of the UC can be obtained by a complete enumeration of all feasible combination of generating units, which could be very huge number $[1,2]$. 
The problem is structured binary variables making it clearly nonconvex. The binary variables cause a great deal of trouble and reason for the difficulty in solving the UC. Load balance constraint and spinning reserve constraint are coupling constraints across the unit so that one unit affects what will happen on other units if the coupling constraints are met. There have been various methods which are based on mathematical programming and metaheuristic-based for solving the thermal and hydrothermal UC problem in literature. They are based on mathematical programming and metaheuristic based approaches. These major methods are priority list [3-5], dynamic programming (DP) [6], mixed-integer programming [7-9], heuristic unit [10], simulated annealing [11-13], tabu search [14, 15], evolutionary programming [16, 17], constraint logic programming [18], genetic algorithms [19-23], LR [24-32], interior point method [33], memetic algorithm [34], and neural network [35-37].

The LR which is one of the most successful approaches for UC is dual optimization technique. This method obtains an appropriate condition to generate feasible solution for UC. One of the most obvious advantages of the LR method is its quantitative measure of the solution quality since the cost of the dual function is a lower bound on the cost of the primal problem [29]. It is seen that LR methods give better solutions than the metaheuristic approaches mentioned above due to both duration time and feasible solution [24-32].

The LR, PF, and ALPF methods which present the solving approach based on dual optimization technique are used for solving the UC problem known as an important and hard-solving problem in power system engineering, and the results from the programs coded and implemented using GAMS for these methods are compared to each other. The UC problem for the four-unit Tuncbilek thermal plant is considered for this analysis. A 24hours day is subdivided into eight discrete stages, and the predicted load of the system can be considered constant over each interval. In this case, the UC problem is an economic combinatorial problem of which solution will yield the minimum operating cost for the scheduling of startup and shutdown of units for each stage in a day.

The remaining sections are outlined as follows. Section 2 provides a description of the UC problem formulation. This section includes an objective function and the constraints of the problem. In Section 3, three methods based on dual optimization techniques are described. In Section 4, the solving algorithm of LR method for the UC problem is explained as a sample. In Section 5, applications and numerical results are presented and discussed. Finally the conclusion is given.

\section{UC Problem Formulation}

UC has been used to plan over a given time horizon the most economical schedule of committing and dispatching generating units to meet forecasted demand levels and spinning reserve requirements while all generating unit constraints are satisfied. The objective function of the UC problem can be formulated as

$$
F\left(P_{i}^{t}, U_{i}^{t}\right)=\sum_{t=1}^{T} \sum_{i=1}^{N}\left[F_{i}\left(P_{i}^{t}\right)+\mathrm{SC}_{i}^{t}\left(1-U_{i}^{t-1}\right)\right] U_{i}^{t}+\sum_{t=1}^{T} \sum_{i=1}^{N} \mathrm{SD}_{i}^{t} x\left(1-U_{i}^{t-1}\right) U_{i}^{t-1}
$$

where $F_{i}\left(P_{i}^{t}\right)=a\left(P_{i}^{t}\right)^{2}+b P_{i}^{t}+c$ is the polynomial function [38]. 
The minimization of the objective function is provided to the following constraints.

\section{System Constraints}

Power balance constraints. For satisfying the load balance in each stage, the forecasted load demand should be equal to the total power of the generated power for feasible combination:

$$
\sum_{i=1}^{N} U_{i}^{t} P_{i}^{t}=P_{d}^{t}
$$

Spinning reserve constraints. In order to meet any abnormal operating conditions, the spinning reserve must be considered:

$$
P_{d}^{t}+P_{r}^{t}-\sum_{i=1}^{N} P_{i, \max } U_{i}^{t} \leq 0
$$

\section{Unit Constraints}

Generation limits. Each unit must satisfy the generation range:

$$
P_{i, \min } U_{i}^{t} \leq P_{i}^{t} \leq P_{i, \max } U_{i}^{t}, \quad i=1,2, \ldots, N
$$

Minimum up-time. Once the unit is started up, it should not be shut down before a minimum up-time period is met as follows:

$$
\left(T_{i}^{\mathrm{on}(t-1)}-T_{i, \mathrm{up}}^{t}\right)\left(U_{i}^{t-1}-U_{i}^{t}\right) \geq 0 .
$$

Minimum down-time. Once the unit is shut down, there is a minimum down time before it can be started up again:

$$
\left(T_{i}^{\text {off }(t-1)}-T_{i, \text { down }}^{t}\right)\left(U_{i}^{t}-U_{i}^{t-1}\right) \geq 0 .
$$

\section{Methods Based on Dual Optimization Technique}

In this paper, three different methods based on dual optimization techniques, LR, PF, and ALPF are used for solving the UC problem. Duality is particularly important in optimization theory. The convergence of the dual optimization methods can be measured by the relative size of the duality gap between the primal and dual solutions. As illustrated in Figure 1, duality gap is obtained by the difference between solutions of primal and dual subproblems. However, primal cost and dual cost functions in reality are not as smooth as the ones in Figure 1 because they are nondifferentiable and nonconvex [39]. 


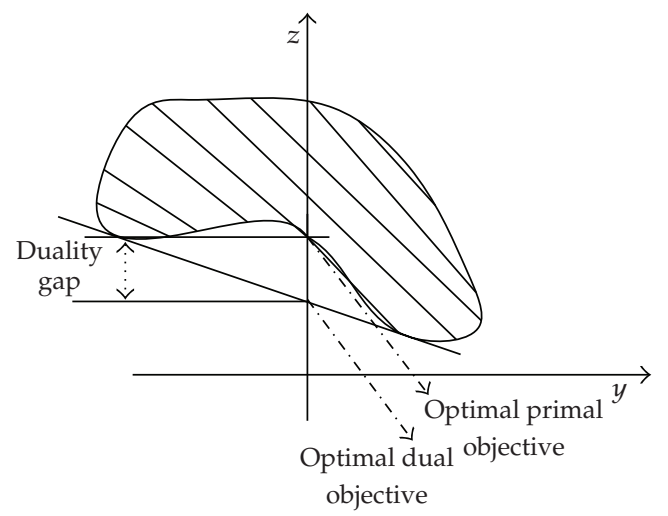

Figure 1: A simplified graphic representation of dual optimization [39].

General constrained minimization problem is as follows:

Primal Problem $(P): \longrightarrow$ Dual Problem: $\left(P^{*}\right)$,

Minimize $f(x) \longrightarrow \max \theta(u)$

Subject to

$$
\begin{aligned}
& g(x) \leq 0 \longrightarrow u>0, \\
& h(x)=0 \longrightarrow u>0 .
\end{aligned}
$$

The dual procedure attempts to reach the constrained optimum by maximizing the Lagrangian with respect to Lagragian multipliers, while minimizing with respect to the other variables in the problem.

\section{LR Method}

LR method can eliminate the dimensionality problem. The LR method is based on the dual optimization theory. The solution of the LR method greatly depends on the Lagragian multipliers. Therefore, setting the initial Lagragian multipliers and updating them are significant to the optimality of the solution. Inappropriate method of updating the Lagrangain multipliers may cause the solution adjustment to oscillate around the global optimum [39].

The Lagrangian equation is

$$
L(x, \lambda, \mu)=f(x)+\sum \lambda_{i} g_{i}(x)+\sum \mu_{i} h_{i}(x) .
$$

Dual function is

$$
q^{*}(\lambda)=\max q(\lambda),
$$


where

$$
q(\lambda)=\min L(x, \lambda, \mu)
$$

The algorithm of LR method is

Choose starting $\lambda, \mu$ values.

Step 1. Find a value for each $\lambda$ and $\mu$ which moves $q(\lambda)$ toward a larger value.

Step 2. Assuming that the $\lambda$ and $\mu$ found in Step 1 are now fixed, find the minimum of the $L$.

\section{PF Method}

PF method is one of the widely used methods for obtaining optimal solutions nonconvex problems. It is motivated by the desire to use unconstrained optimization techniques to solve constrained problems. It uses a mathematical function that will increase the objective for any given constrained violation. General transformation of constrained problem into an unconstrained problem [39]

$$
\begin{gathered}
L(x, k, M)=f_{0}(x)+k \sum_{i=1}^{n}\left[\operatorname{maks}\left\{0, g_{i}(x)\right\}\right]^{2}+M \sum_{j=1}^{m}\left|h_{j}(x)\right|, \\
f_{0}(x)+\phi_{1}(g(x), k)+\phi_{2}(h(x), M) .
\end{gathered}
$$

The algorithm of PF method is

Choose $\varepsilon>0$ and $\beta>1$. Choose initial value $x_{1}$.

Choose penalty factor $M_{1}$.

Step 3. Find the following values:

$$
\begin{gathered}
\operatorname{Min}\left\{f_{0}(x)+M_{1}[\operatorname{maks}\{0, g(x)\}]\right\}, \\
\operatorname{Min}\left\{f_{0}(x)+M_{k} \alpha x\right\}=f_{0}\left(x_{k}\right)+M_{k} \alpha x_{k} .
\end{gathered}
$$

Step 4. If $M_{k} \alpha x_{k}<\varepsilon=0$, then stop. Otherwise, $M_{k+1}=\beta M_{k}, k=k+1$. Then go to Step 1 .

\section{ALPF Method}

The ALPF method is an approach for solving nonlinear programming problems by using the ALPF in a manner that combines the algorithmic aspects of both LR and PF methods [39].

Lagragian equation is

$$
L(x, c, v, M)=f_{0}(x)+\sum_{i=1}^{m} v_{i} g_{i}(x)+\sum_{i=1}^{q} v_{j} h_{j}(x)+M_{i}\left[\sum_{i=1}^{m} g_{i}(x)\right]^{2}+M_{j}\left[\sum_{i=1}^{q} h_{j}(x)^{2}\right]
$$


The algorithm of ALPF method is as follows.

\section{Initialization}

Select some initial Lagrangian multipliers. Lagrangian multipliers are $v^{m}$ and $M^{m}$. These are can be written as follows:

$$
\begin{aligned}
v^{m} & =\left(v_{1}^{1}, v_{2}^{1}, \ldots, v_{m}^{1}\right), \\
M^{m} & =\left(M_{1}^{1}, M_{2}^{1}, \ldots, M_{m}^{1}\right),
\end{aligned}
$$

where $v^{m}$ is positive values, and $M^{m}$ is for the penalty parameters.

Let $x_{0}$ be a null vector and denote

$$
\operatorname{VIOL}\left(x_{0}\right)=+\infty
$$

for any $x \in E_{n}$.

Measure the level of the violation by using (3.7)

$$
\operatorname{VIOL}(x)=\max _{1 \leq i \leq m}\left\{\left|f_{i}(x)\right|\right\}
$$

Choose $k=1 ; \varepsilon>0$ is a measure of constraint violations. Put $k=1$, and proceed to the "inner loop" of the algorithm.

\section{Inner Loop}

Solve minimum of the Lagrangian subject to $x \in E_{n}$, and let $x_{k}$ be the optimal solution obtained. If $\operatorname{VIOL}\left(x_{k}\right)=0$ or $\operatorname{VIOL}\left(x_{k}\right)<\varepsilon$, then stop. Otherwise, if $\operatorname{VIOL}\left(x_{k}\right)<$ $(1 / 4) \operatorname{VIOL}\left(x_{k-1}\right)$, then go to outer loop. If $\operatorname{VIOL}\left(x_{k}\right)>\operatorname{VIOL}\left(x_{k-1}\right)$, then, for each constraint $i=1, \ldots, l$ for which $\left\|f_{i}\left(x_{k}\right)\right\|>(1 / 4) \operatorname{VIOL}\left(x_{k-1}\right)$, replace the corresponding penalty parameter $M_{i}$ by $10 M_{i}$ and repeat this inner loop step.

\section{Outer Loop}

Replace $v$ by $v_{\text {new }}$, where

$$
v_{i}^{k+1}=v_{i}^{k}+2 M_{i}^{k} f_{i}\left(x_{k}\right) .
$$

Increment $k$ by 1 , and return to the inner loop.

\section{Solving Algorithm for UC}

The LR method solves the UC problem by relaxing or temporarily ignoring the coupling constraints. This is done through the dual optimization procedure attempting to reach the constrained optimum by maximizing the Lagrangian. In this section, the solving algorithm 
of LR method for the UC problem is explained as a sample. The Lagrangian equation can be written as follows [40]:

$$
\begin{aligned}
L(P, U, \lambda)= & \sum_{t=1}^{T} \sum_{i=1}^{N} F\left(P_{i}^{t}, U_{i}^{t}\right)+\mathrm{SC}_{i}^{t}\left(1-U_{i}^{t-1}\right) U_{i}^{t}+\sum_{t=1}^{T} \sum_{i=1}^{N} \mathrm{SD}_{i}^{t} x\left(1-U_{i}^{t-1}\right) \\
& +\sum_{t=1}^{T} \lambda^{t}\left(P_{\text {load }}^{t}-\sum_{i=1}^{N} P_{i}^{t} U_{i}^{t}\right)+\sum_{t=1}^{T} \mu^{t}\left(P_{\text {load }}^{t}+P_{r}(t)-\sum_{i=1}^{N} P_{i, \max } U_{i}^{t}\right) .
\end{aligned}
$$

$q^{*}(\lambda, \mu)$ is a dual function, and it can be shown as follows:

$$
q^{*}(\lambda, \mu)=\max _{\lambda^{t}, \mu^{t}} q(\lambda, \mu)
$$

where

$$
\begin{aligned}
q(\lambda, \mu)= & \min _{P_{i}^{t}, U_{i, t}} L(P, U, \lambda, \mu), \\
L= & \sum_{t=1}^{T} \sum_{i=1}^{N}\left[F\left(P_{i}^{t}, U_{i}^{t}\right)+\mathrm{SC}_{i}^{t}\left(1-U_{i}^{t-1}\right) U_{i}^{t}\right]+\sum_{t=1}^{T} \sum_{i=1}^{N} \operatorname{SD}_{i}^{t} x\left(1-U_{i}^{t-1}\right) \\
& +\sum_{t=1}^{T} \lambda^{t} P_{\text {load }}^{t}+\mu^{t}\left(P_{\text {load }}^{t}+P_{r}(t)\right)-\sum_{i=1}^{N} \lambda^{t} P_{i}^{t} U_{i}^{t}-\mu^{t} P_{i, \max } U_{i}^{t},
\end{aligned}
$$

where $\lambda^{t} P_{\text {load }}^{t}$, and $\mu^{t}\left(P_{\text {load }}^{t}+P_{r}(t)\right)$ are constant and can be dropped.

Goal of separating the units from one another has been achieved. The term inside the outer brackets

$$
\begin{aligned}
\sum_{t=1}^{T}\{ & {\left.\left[F\left(P_{i}^{t}, U_{i}^{t}\right)+\mathrm{SC}_{i}^{t}\left(1-U_{i}^{t-1}\right) U_{i}^{t}+\sum_{t=1}^{T} \sum_{i=1}^{N} \mathrm{SD}_{i}^{t} x\left(1-U_{i}^{t-1}\right)\right]\right\} } \\
& -\lambda^{t} P_{i}^{t} U_{i}^{t-1}-\mu^{t} P_{i, \max } U_{i, t} .
\end{aligned}
$$

Equation (4.4) can be solved separately for each generating unit, without regard for what is happening on the other generating units. The minimum of the Lagrangian is found by solving for the minimum for each generating unit over all time periods

$$
\begin{aligned}
\min q(\lambda)= & \sum_{i=1}^{N} \min \sum_{t=1}^{T}\left\{\left[F\left(P_{i}^{t}, U_{i}^{t}\right)+\mathrm{SC}_{i}^{t}\left(1-U_{i}^{t-1}\right) U_{i}^{t}+\sum_{t=1}^{T} \sum_{i=1}^{N} \mathrm{SD}_{i}^{t} x\left(1-U_{i}^{t-1}\right)\right]\right\} \\
& -\lambda^{t} P_{i}^{t} U_{i}^{t}-\mu^{t} P_{i, \max } U_{i}^{t-1} .
\end{aligned}
$$

This is solved as a one-variable DP problem. This can be visualized in Figure 2, which shows the only two possible states for unit $i$. 


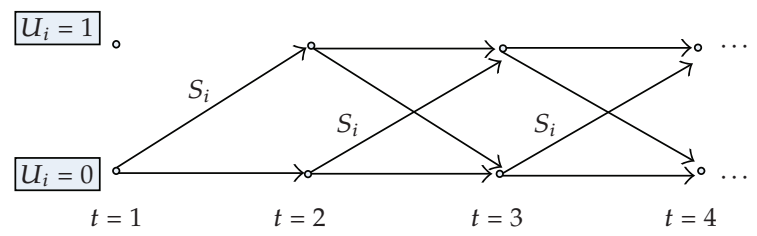

Figure 2: Only two possible states for unit $i\left(U_{i}^{t}=0\right.$ or $\left.U_{i}^{t}=1\right)$, where $S_{i}$ is the startup cost for unit $i$.

At the $U_{i, t}=0$ state, the value of the function to be minimized is trivial. At the state $U_{i, t}=1$, the function to be minimized is.

$$
\min \left[F_{i} P_{i}^{t}-\lambda^{t} P_{i}^{t}-\mu^{t} P_{i, \max }\right]
$$

The minimum of this function is

$$
\frac{d}{d P_{i}^{t}}\left[F_{i} P_{i}^{t}-\lambda^{t} P_{i}^{t}-\mu^{t} P_{i, \max }\right]=0
$$

The solution to this equation is

$$
\begin{gathered}
\frac{d F_{i}\left(P_{i}^{t, \mathrm{opt}}\right)}{d P_{i}^{t}}=\lambda^{t}, \\
P_{i}^{t, \mathrm{opt}}=\frac{\lambda^{t}-b_{i}}{2 c_{i}} .
\end{gathered}
$$

There are three cases in this situation.

(1) If $P_{i}^{t, \text { opt }}<P_{i, \text { min }}$ then $P_{i}^{t}=P_{i, \min }$.

(2) If $P_{i, \min }<P_{i}^{t, \text { opt }}<P_{i, \max }$, then $P_{i}^{t}=P_{i}^{t, \text { opt }}$.

(3) If $P_{i}^{t, \text { opt }}>P_{i, \text { max }}$ then $P_{i}^{t}=P_{i, \max }$.

DP is used to determine the optimal schedule of each unit over the scheduled time period. To minimize $F\left(P_{i}^{t}\right)-\lambda^{t} P_{i}^{t}<0$ at each stage and that when $U_{i}^{t}=0$, this value goes to zero, then the only way to get a value lower is to have [40]

$$
F\left(P_{i}^{t}\right)-\lambda^{t} P_{i}^{t}<0
$$

In the LR method, the value obtained by (2.1) and problem constraints is called the primal value $\left(J^{*}\right)$ of the problem. The value in (3.3) represents the dual value. Whether the problem converges to its solution is decided by looking at the relative duality gap value. This value is calculated using (4.10)

$$
\text { Relative duality gap }=\frac{J^{*}-q^{*}}{q^{*}} \text {. }
$$


Table 1: Unit characteristics for four-unit Tuncbilek thermal plant.

\begin{tabular}{lccccccccc}
\hline Unit & $P_{\min }(\mathrm{MW})$ & $P_{\max }(\mathrm{MW})$ & $\mathrm{SC}(\$)$ & $\mathrm{a}(\$ / \mathrm{h})$ & $\mathrm{b}(\$ / \mathrm{MWh})$ & $\mathrm{c}\left(\$ / \mathrm{MW}^{2} \mathrm{~h}\right)$ & $\mathrm{SD}(\$)$ & $T_{\text {up }}(\mathrm{h})$ & $T_{\text {down }}(\mathrm{h})$ \\
\hline 1 & 8 & 32 & 60 & 0.515 & 10.86 & 149.9 & 120 & 1 & 1 \\
2 & 17 & 65 & 240 & 0.227 & 8.341 & 284.6 & 480 & 2 & 2 \\
3 & 35 & 150 & 550 & 0.082 & 9.9441 & 495.8 & 1100 & 3 & 3 \\
4 & 30 & 150 & 550 & 0.074 & 12.44 & 388.9 & 1100 & 3 & 3 \\
\hline
\end{tabular}

Table 2: Load data (MW).

\begin{tabular}{lccc}
\hline Stage & Load & Stage & Load \\
\hline 1 & 168 & 5 & 313 \\
2 & 150 & 6 & 347 \\
3 & 260 & 7 & 308 \\
4 & 275 & 8 & 231 \\
\hline
\end{tabular}

Table 3: Feasible unit combination for LR method.

\begin{tabular}{|c|c|}
\hline Stage & Unit combination \\
\hline 1 & 0011 \\
\hline 2 & 0011 \\
\hline 3 & 0111 \\
\hline 4 & 1111 \\
\hline 5 & 1111 \\
\hline 6 & 1111 \\
\hline 7 & 1111 \\
\hline 8 & 0111 \\
\hline
\end{tabular}

\section{Applications and Numerical Results}

The LR, PF, and ALPF methods are applied for solving the UC problem for four-unit Tuncbilek thermal plant separately, and the numerical results are given in tables. These methods are compared to each other based on feasible schedule for each stage, feasible cost, dual cost, duality gap, duration time, and number of iterations using the table in the comparative results part. The spinning reserve demand is set as $10 \%$ of the system demand. All these methods are coded in GAMS which is a high-level modeling system for mathematical programming problems [41] and run on Pentium 4-3 GHz personal computer. CONOPT is used as a GAMS solver. The data used in the analysis is taken from Turkish Electric Power Company and Electricity Generation Company. The unit characteristics for four-unit Tuncbilek thermal plant are given in Table 1.

In this study, a 24-hours day is subdivided into 8 discrete stages. The load demands for the stages are given in Table 2.

\section{For LR Method}

LR method is applied to UC problem. The feasible unit combination for the LR method is given in Table 3 . The initial of the $\lambda$ and $\mu$ values is chosen as zero. 
Table 4: Feasible unit combination for PF method.

\begin{tabular}{|c|c|}
\hline Stage & Unit combination \\
\hline 1 & 0011 \\
\hline 2 & 0011 \\
\hline 3 & 0111 \\
\hline 4 & 0111 \\
\hline 5 & 1111 \\
\hline 6 & 1111 \\
\hline 7 & 0111 \\
\hline 8 & 0111 \\
\hline
\end{tabular}

Table 5: Feasible unit combination for ALPF method.

\begin{tabular}{|c|c|}
\hline Stage & Unit combination \\
\hline 1 & 0011 \\
\hline 2 & 0011 \\
\hline 3 & 0111 \\
\hline 4 & 0111 \\
\hline 5 & 0111 \\
\hline 6 & 1111 \\
\hline 7 & 0111 \\
\hline 8 & 0111 \\
\hline
\end{tabular}

Table 6: The numerical results for LR, PF, and ALPF methods.

\begin{tabular}{lccc}
\hline Compared items & LR & PF & ALPF \\
\hline Feasible cost $(\$)$ & 56381.931 & 55801.477 & 55233.897 \\
Dual cost $(\$)$ & 55428.014 & 55305.332 & 54894.266 \\
Duality gap $(\%)$ & 1.1721 & 0.8971 & 0.6187 \\
Number of iterations & 19 & 36 & 67 \\
Duration time $(s)$ & 5.32 & 3.56 & 14.63 \\
\hline
\end{tabular}

\section{For PF Method}

PF method is applied to UC problem. The feasible unit combination for the PF method is given in Table 4 . The initial of the $k$ and $M$ values are 320 and 970, respectively.

\section{For ALPF Method}

ALPF method is applied to UC problem. The feasible unit combination for the ALPF method is given in Table 5. The initial of the $v$ and $M$ values is 25 and 100, respectively.

\section{Comparative Results}

Feasible cost, dual cost and duality gap values, duration time, and number of iterations are found for each method. These numerical results are given in Table 6.

It can be seen from Table 6 that LR has the highest duality gap value, and ALPF performs much better than LR and PF for duality gap value. It is noticed that ALPF method yields the minimum duality gap value with minimum cost. On the other hand, LR method 
reaches the solution with the minimum number of iterations. In addition to this, PF method yields the fastest solution.

\section{Conclusion}

Three different dual approach-based methods, LR, PF and ALPF methods, are used for solving the UC problem known as an important and hard-solving problem in power system engineering, and the results from the programs coded and implemented using GAMS for these methods are compared to each other according to feasible cost, dual cost, duality gap, duration time, and number of iterations. The numerical results show that the ALPF method which is firstly used for solving the UC problem in literature gives the best duality gap, feasible and dual cost values instead of worse duration time and the number of iterations. It is seen that ALPF method yields the minimum duality gap value with minimum cost. On the other hand, LR method reaches the solution with the minimum number of iterations. In addition to this, PF method yields the fastest solution. Since the most important factor in the solution of UC problems is feasible cost value, it can be said that ALPF is the most suitable method among others.

\section{List of symbols}

$f(x)$ : Objective function

$\bar{H}$ : $\quad$ Upper bound of the dual function

$h(x)$ : Equality constraint

$g(x)$ : Inequality constraint

$k$ : $\quad$ Multiplier for PF method

M: $\quad$ Multiplier for PF and ALPF method

$N$ : $\quad$ Number of generating units

$P: \quad$ Primal problem

$P^{*}: \quad$ Dual problem

$P_{d}^{t}: \quad$ Nominal demand at hour $t$ (MW)

$P_{i}^{t}: \quad$ Generation output of unit $i$ at hour $t$ (MW)

$P_{\text {max }, i}^{t}$ : Maximum available capacity of unit $i$ at hour $t$ (MW)

$P_{\min , i}^{t}:$ Minimum available capacity of unit $i$ at hour $t$ (MW)

$P_{r}^{t}: \quad$ System spinning reserve at hour $t(\mathrm{MW})$

$\mathrm{RU}_{i}: \quad$ Ramp up rate of unit $i(\mathrm{MW} / \mathrm{h})$

$\mathrm{RD}_{i}$ : $\quad$ Ramp down rate of unit $i(\mathrm{MW} / \mathrm{h})$

$\mathrm{SC}_{i}: \quad$ Start up cost of unit $i(\$)$

$\mathrm{SD}_{i}: \quad$ Shut down cost of unit $i(\$)$

$U_{i}^{t}$ : Status value of unit $i$ at time $t$

$T: \quad$ Time horizon for UC (h)

$T_{i, \text { down }}$ : Minimum down-time of unit $i, \mathrm{~h}$

$T_{i, \text { off }}^{t}:$ Continuously off-time of unit $i$ up to hour $t, \mathrm{~h}$

$T_{i, \text { on }}^{t}$ : Continuously on-time of unit $i$ up to hour $t, \mathrm{~h}$

$T_{i, \text { down }}$ : Minimum up-time of unit $i, \mathrm{~h}$

TC: $\quad$ Total cost (\$)

v: $\quad$ Multiplier for ALPF. 


\section{References}

[1] A. H. Mantawy, Y. L. Abdel-Magid, and S. Z. Selim, "A simulated annealing algorithm for unit commitment," IEEE Transactions on Power Systems, vol. 13, no. 1, pp. 197-204, 1998.

[2] A. Viana, J. P. Sousa, and M. Matos, "Simulated annealing for the unit commitment problem," in Proceedings of IEEE Porto Power Tech Conference, vol. 2, pp. 1-4, Porto, Portugal, September 2001.

[3] F. N. Lee and Q. Feng, "Multi-area unit commitment," IEEE Transactions on Power Systems, vol. 7, no. 2, pp. 591-599, 1992.

[4] F. N. Lee, "The application of commitment utilization factor (CUF) to thermal unit commitment," IEEE Transactions on Power Systems, vol. 6, no. 2, pp. 691-698, 1991.

[5] R. M. Burns and C. A. Gibson, "Optimization of priority lists for a unit commitment program," in Proceedings of IEEE/PES Summer Meeting, San Francisco, Calif, USA, July 1975, paper A 75 453-1.

[6] C.-L. Chen and N. Chen, "Strategies to improve the dynamic programming for unit commitment application," Transactions of the Chinese Institute of Engineers, vol. 9, no. 3, pp. 181-189, 2002.

[7] J. M. Arroyo and A. J. Conejo, "Optimal response of a thermal unit to an electricity spot market," IEEE Transactions on Power Systems, vol. 15, no. 3, pp. 1098-1104, 2000.

[8] X. Guan, Q. Zhai, and A. Papalexopoulos, "Optimization based methods for unit commitment: Lagrangian relaxation versus general mixed integer programming," in Proceedings of IEEE/PES Summer Meeting, vol. 2, pp. 1095-1100, Toronto, Canada, July 2003.

[9] T. Li and M. Shahidehpour, "Price-based unit commitment: a case of Lagrangian relaxation versus mixed integer programming," IEEE Transactions on Power Systems, vol. 20, no. 4, pp. 2015-2025, 2005.

[10] U. D. Annakkage, T. Nummonda, and N. C. Pahalawaththa, “Unit commitment by parallel simulated annealing," IEE Proceedings: Generation, Transmission and Distribution, vol. 142, no. 6, pp. 595-600, 1995.

[11] R. Nayak and J. D. Sharma, "Hybrid neural network and simulated annealing approach to the unit commitment problem," Computers and Electrical Engineering, vol. 26, no. 6, pp. 461-477, 2000.

[12] C. C. A. Rajan, M. R. Mohan, and K. Manivannan, "Refined simulated annealing method for solving unit commitment problem," in Proceedings of the International Joint Conference on Neural Networks (IJCNN '02), vol. 1, pp. 333-338, Honolulu, Hawaii, USA, May 2002.

[13] A. H. Mantawy, Y. L. Abdel-Magid, and S. Z. Selim, "A simulated annealing algorithm for unit commitment," IEEE Transactions on Power Systems, vol. 13, no. 1, pp. 197-204, 1998.

[14] C. C. A. Rajan, M. R. Mohan, and K. Manivannan, "Neural based Tabu search method for solving unit commitment problem," in Proceedings of the 5th International Conference on Power System Management and Control, pp. 180-185, London, UK, April 2002.

[15] H. Mori and T. Usami, "Unit commitment using Tabu search with restricted neighborhood," in Proceedings of the International Conference on Intelligent Systems Applications to Power Systems (ISAP '96), pp. 422-427, Orlando, Fla, USA, January-February 1996.

[16] C. C. A. Rajan and M. R. Mohan, "An evolutionary programming-based Tabu search method for solving the unit commitment problem," IEEE Transactions on Power Systems, vol. 19, no. 1, pp. 577$585,2004$.

[17] K. A. Juste, H. Kita, E. Tanaka, and J. Hasegawa, “An evolutionary programming solution to the unit commitment problem," IEEE Transactions on Power Systems, vol. 14, no. 4, pp. 1452-1459, 1999.

[18] K.-Y. Huang, H.-T. Yang, and C.-L. Huang, "A new thermal unit commitment approach using constraint logic programming," IEEE Transactions on Power Systems, vol. 13, no. 3, pp. 936-945, 1998.

[19] K. S. Swarup and S. Yamashiro, "Unit commitment solution methodology using genetic algorithm," IEEE Transactions on Power Systems, vol. 17, no. 1, pp. 87-91, 2002.

[20] N. P. Padhy, "Unit commitment using hybrid models: a comparative study for dynamic programming, expert system, fuzzy system and genetic algorithms," International Journal of Electrical Power $\mathcal{E}$ Energy Systems, vol. 23, no. 8, pp. 827-836, 2001.

[21] T. Senjyu, H. Yamashiro, K. Uezato, and T. Funabashi, "A unit commitment problem by using genetic algorithm based on unit characteristic classification," in Proceedings of IEEE/PES Winter Meeting, vol. 1, pp. 58-63, Singapore, January 2002.

[22] P.-C. Yang, H.-T. Yang, and C.-L. Huang, "Solving the unit commitment problem with a genetic algorithm through a constraint satisfaction technique," Electric Power Systems Research, vol. 37, no. 1, pp. 55-65, 1996.

[23] S. A. Kazarlis, A. G. Bakirtzis, and V. Petridis, "A genetic algorithm solution to the unit commitment problem," IEEE Transactions on Power Systems, vol. 11, no. 1, pp. 83-92, 1996.

[24] R. Nieva, A. Inda, and I. Guillen, "Lagrangian reduction of search-range for large-scale unit commitment," IEEE Transactions on Power Systems, vol. 2, no. 2, pp. 465-473, 1987. 
[25] S. Virmani, E. C. Adrian, K. Imhof, and S. Mukherjee, "Implementation of a Lagrangian relaxation based unit commitment problem," IEEE Transactions on Power Systems, vol. 4, no. 4, pp. 1373-1380, 1989.

[26] J. Batut and A. Renaud, "Daily generation scheduling optimization with transmission constraints: a new class of algorithms," IEEE Transactions on Power Systems, vol. 7, no. 3, pp. 982-989, 1992.

[27] S. J. Wang, S. M. Shahidehpour, D. S. Kirschen, S. Mokhtari, and G. D. Irisarri, "Short-term generation scheduling with transmission and environmental constraints using an augmented Lagrangian relaxation," IEEE Transactions on Power Systems, vol. 10, no. 3, pp. 1294-1301, 1995.

[28] N. J. Redondo and A. J. Conejo, "Short-term hydro-thermal coordination by Lagrangian relaxation: solution of the dual problem," IEEE Transactions on Power Systems, vol. 14, no. 1, pp. 89-95, 1999.

[29] Q. Zhai, X. Guan, and J. Cui, “Unit commitment with identical units: successive subproblem solving method based on Lagrangian relaxation," IEEE Transactions on Power Systems, vol. 17, no. 4, pp. 12501257, 2002.

[30] W. Ongsakul and N. Petcharaks, "Unit commitment by enhanced adaptive Lagrangian relaxation," IEEE Transactions on Power Systems, vol. 19, no. 1, pp. 620-628, 2004.

[31] P. Sriyanyong and Y. H. Song, "Unit commitment using particle swarm optimization combined with Lagrange relaxation," in Proceedings of IEEE/PES General Meeting, vol. 3, pp. 2752-2759, San Francisco, Calif, USA, June 2005.

[32] Y. Fu, M. Shahidehpour, and Z. Li, "Long-term security-constrained unit commitment: hybrid Dantzig-Wolfe decomposition and subgradient approach," IEEE Transactions on Power Systems, vol. 20, no. 4, pp. 2093-2106, 2005.

[33] L. M. Kimball, K. A. Clements, P. W. Davis, and I. Nejdawi, "Multiperiod hydrothermal economic dispatch by an interior point method," Mathematical Problems in Engineering, vol. 8, no. 1, pp. 33-42, 2002.

[34] J. Valenzuela and A. E. Smith, "A seeded memetic algorithm for large unit commitment problems," Journal of Heuristics, vol. 8, no. 2, pp. 173-195, 2002.

[35] H. Sasaki, M. Watanabe, J. Kubokawa, N. Yorino, and R. Yokoyama, "A solution method of unit commitment by artificial neural networks," IEEE Transactions on Power Systems, vol. 7, no. 3, pp. 974981, 1992.

[36] R.-H. Liang and F.-C. Kang, "Thermal generating unit commitment using an extended mean field annealing neural network," IEE Proceedings: Generation, Transmission and Distribution, vol. 147, no. 3, pp. 164-170, 2000.

[37] S.-J. Huang and C.-L. Huang, "Application of genetic-based neural networks to thermal unit commitment," IEEE Transactions on Power Systems, vol. 12, no. 2, pp. 654-660, 1997.

[38] V. N. Dieu and W. Ongsakul, "Improved merit order and augmented Lagrange Hopfield network for unit commitment," IET Generation, Transmission E Distribution, vol. 1, no. 4, pp. 548-556, 2007.

[39] M. S. Bazaraa, H. D. Sherali, and C. M. Shetty, Nonlinear Programming: Theory and Algorithms, John Wiley \& Sons, Hoboken, NJ, USA, 2nd edition, 1993.

[40] A. J. Wood and B. F. Wollenberg, Power Generation Operation and Control, Wiley-Interscience, New York, NY, USA, 2nd edition, 1996.

[41] A. Brooke, D. Kendrick, A. Meeraus, and R. Raman, "GAMS: a user's guide," GAMS Development Corporation, 1998, http:/ /www.gams.com/. 


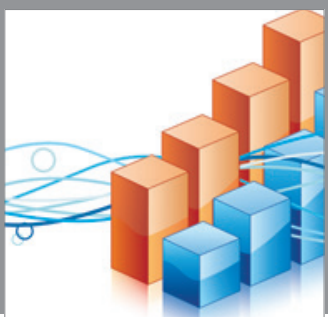

Advances in

Operations Research

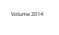

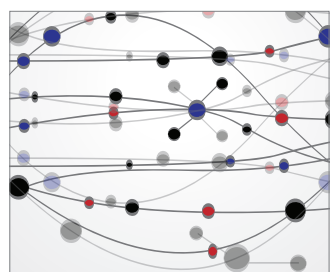

\section{The Scientific} World Journal
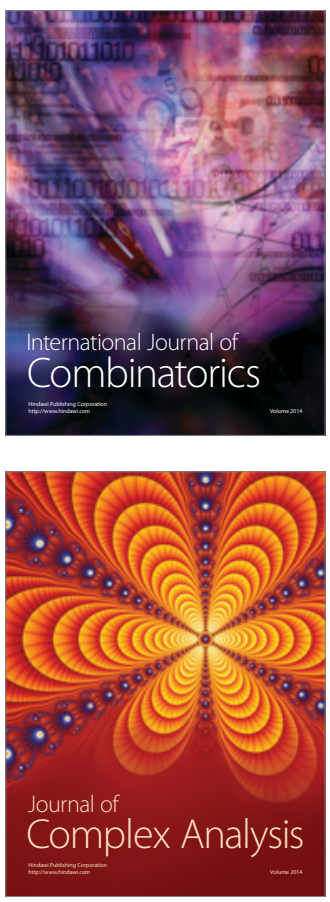

International Journal of

Mathematics and

Mathematical

Sciences
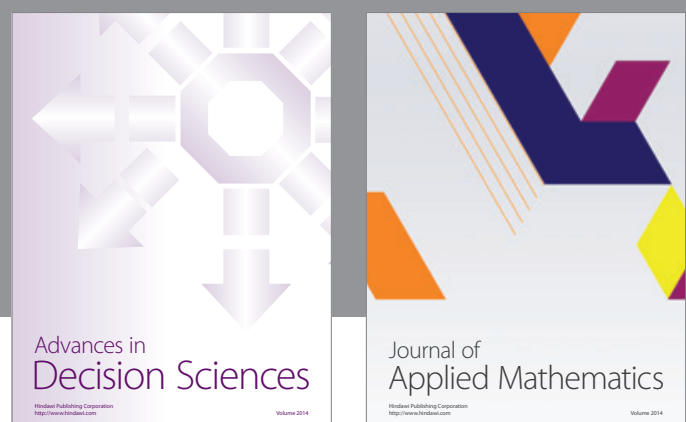

Journal of

Applied Mathematics
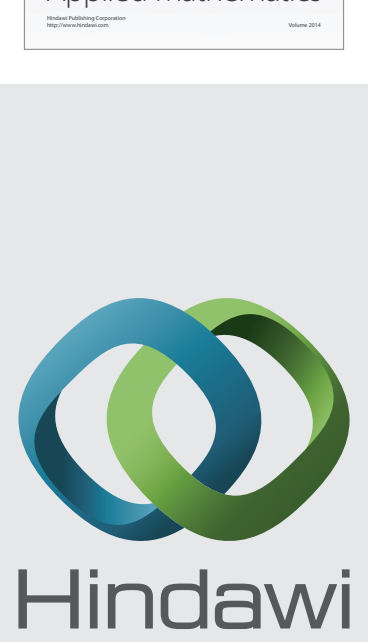

Submit your manuscripts at http://www.hindawi.com
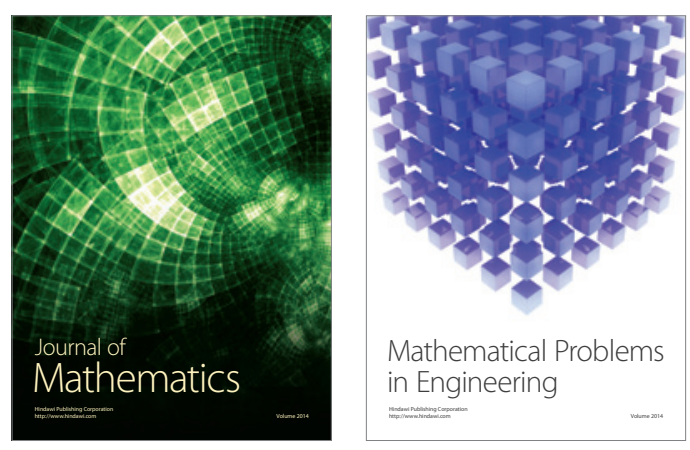

Mathematical Problems in Engineering
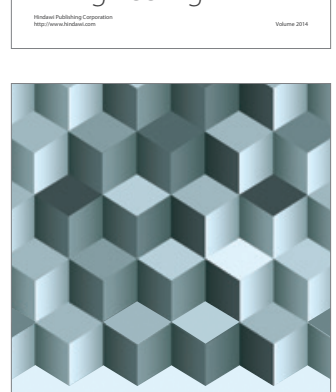

Journal of

Function Spaces
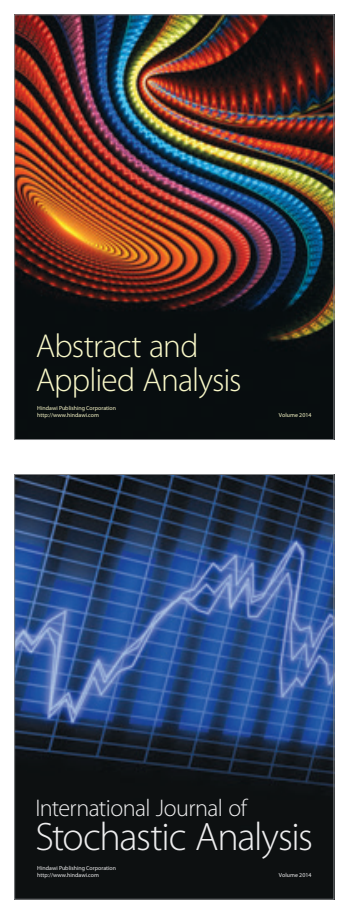

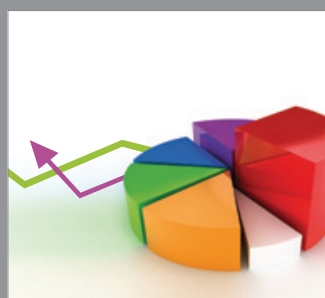

ournal of

Probability and Statistics

Promensencen
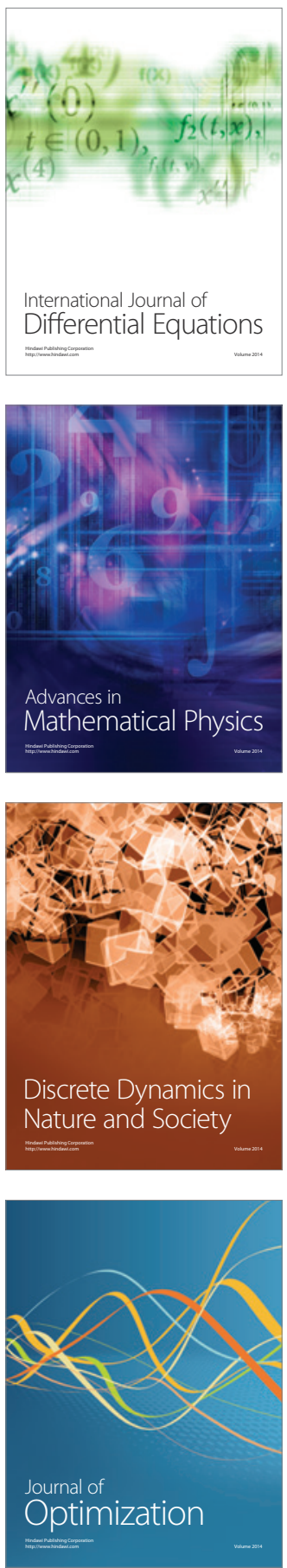